\title{
Bearing capacity and settlement of circular shallow foundations using a non-linear constitutive relationship
}

\author{
Authors: \\ B. T. McMahon, BE (Hons) Syd. \\ Graduate Researcher, Department of Engineering, University of Cambridge \\ Contact Address: $\quad$ Schofield Centre \\ High Cross \\ Madingley Road \\ Cambridge CB3 OEL \\ United Kingdom \\ Corresponding author: b.mcmahon84@gmail.com
}

\begin{abstract}
S. K. Haigh, MA MEng PhD Cantab.
University Lecturer, Department of Engineering, University of Cambridge

Contact email: $\quad \underline{\text { skh20@cam.ac.uk }}$
\end{abstract}
M. D. Bolton, MA MSc PhD Cantab. MICE CEng FREng
Professor of Soil Mechanics, Department of Engineering, University of Cambridge
Contact email: $\quad$ mdb8@cam.ac.uk

Number of words: 4426 (main text); Number of tables: 0; Number of figures: 9

Date version drafted:

$24^{\text {th }}$ November 2013

Page 1 of 26 


\title{
Bearing capacity and settlement of circular shallow foundations using a non-linear constitutive relationship
}

\author{
B. T. McMahon, S. K. Haigh and M. D. Bolton
}

\begin{abstract}
$\underline{\text { Abstract }}$
The design of shallow foundations is dominated by issues of settlement, rather than by bearing capacity per-se. The ability to predict the settlement of foundations at a given factor of safety is hence of key importance in design. In this paper, the energy method for a linear-elastic perfectly plastic method utilizing the von Mises' yield criterion with associated flow developed by McMahon et al. (2013a) is extended to consider the non-linear behaviour of soil. The energy method is used to investigate the load-settlement behaviour of shallow foundations by utilizing an ellipsoidal cavity-expansion mechanism and deformation fields within the boundaries of the classical Hill and Prandtl mechanisms. An elastic mechanism obtained from an analysis in ABAQUS was also investigated using this energy method. The upper-bound approach demonstrates that the cavity-expansion mechanism produces a better solution at small values of settlement, whereas at greater settlements the Prandtl mechanism is shown to produce a more optimal upper-bound solution.
\end{abstract}

$\underline{\text { Keywords: }}$ bearing capacity, clays, foundations; plasticity, settlement 


\section{Page 3 of 35}

\section{NOTATION}

\section{$\underline{\text { Roman }}$}

b empirical exponent

$c_{u} \quad$ undrained shear strength

D footing diameter

F $\quad$ factor of safety

$\mathrm{K}_{0} \quad$ earth pressure coefficient at rest

$N_{c} \quad$ bearing capacity factor

$p \quad$ mean principal stress

$p_{0} \quad$ in-situ stress

$q_{m o b} \quad$ mobilised strength in triaxial compression

$r \quad$ radial coordinate

$r_{b} \quad$ boundary radius of analysis

$r_{f} \quad$ footing radius

$u \quad$ radial displacement

$v \quad$ vertical displacement

W work

$W_{b, n-l} \quad$ non-linear plastic work outside analysis boundary

$W_{n-l} \quad$ non-linear plastic work

$W_{p} \quad$ perfectly plastic work

$z \quad$ depth 


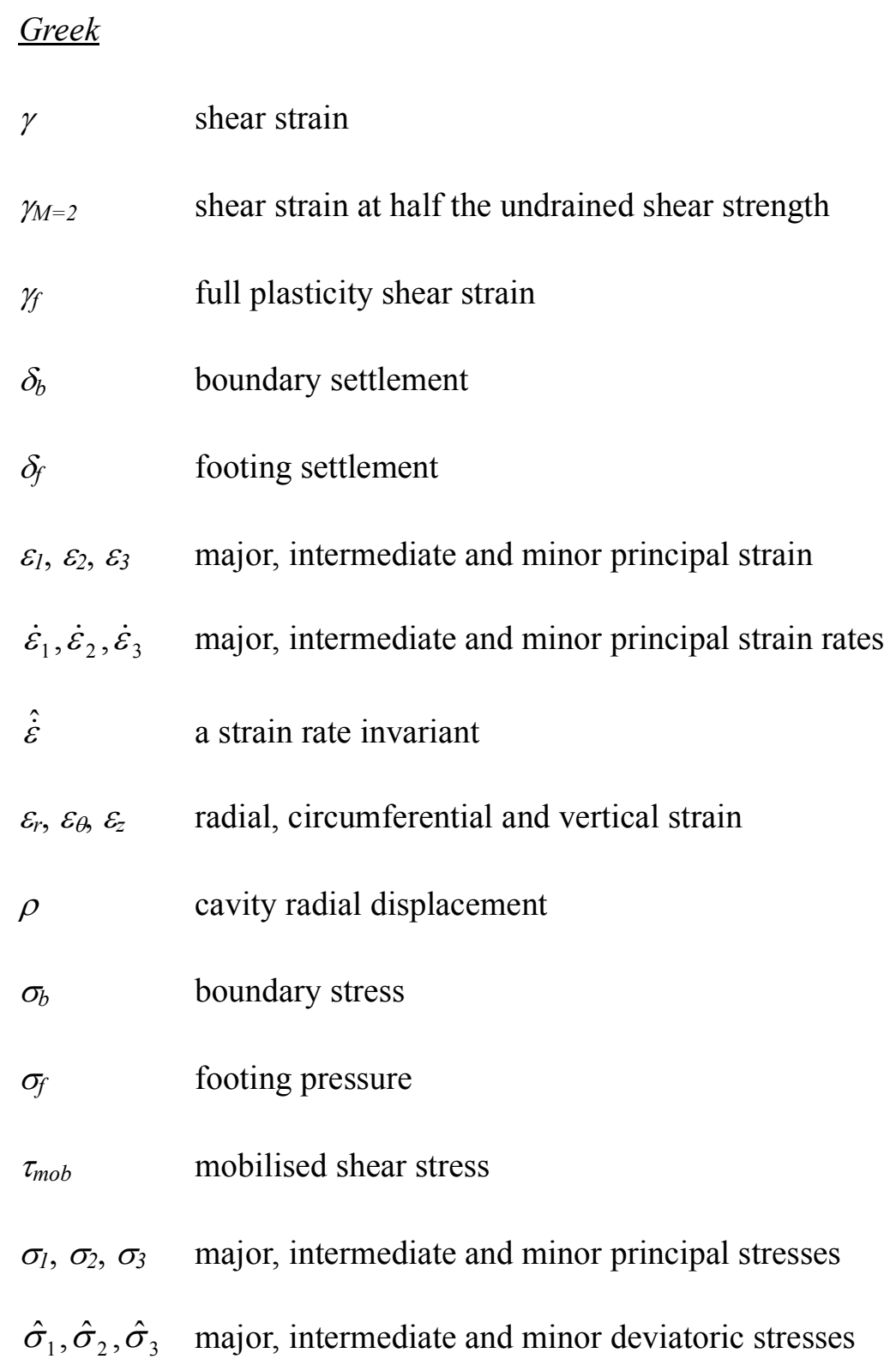




\section{INTRODUCTION}

Shallow foundations are widely used to support lightly loaded structures on clay soils, as well as more heavily loaded structures such as fluid storage tanks. These are typically designed using the classical Prandtl equation:

$$
\sigma_{f}=(2+\pi) c_{u}
$$

where $\sigma_{f}$ is the vertical stress on the foundation and $c_{u}$ is the undrained shear strength of the soil. A factor of safety is then typically used as a reduction factor on the calculated load capacity. The purpose of this factor of safety, as discussed by Bolton (2013) in his Rankine Lecture, is not only to provide a margin of safety but to limit the deformations of the structure being supported. Whereas clays typically mobilise their full strength at a shear strain of the order of $10 \%$, masonry structures would typically begin to show damage if their supporting soils strain by more than $0.1 \%$ : see Figure 1 (Mair, 1993). The use of factors of safety allows settlements and strains to be limited, but the link between factor of safety, soil stiffness and settlement is not explicit in this design approach.

Linear-elasticity of soil is often used to calculate the settlement of shallow foundations. The stress-strain behaviour of soil is, however, highly non-linear even from very small strains (Jardine et al. 1984; Burland, 1989; Houlsby and Wroth, 1991), so the choice of what stiffness to use within an elastic analysis is problematic, the stiffness changing substantially with increasing strain level, as also shown in Figure 1.

Osman and Bolton (2005) considered the non-linear behaviour of soil by scaling a triaxial test stress-strain curve to a footing load-settlement curve in the process of Mobilisable Strength

Page 5 of 26 
Design (MSD). In the development of MSD, a displacement field within the boundary of the Prandtl mechanism was used to determine appropriate scaling factors, in this case a compatibility factor and the well-known bearing capacity factor $N_{c}$. Klar and Osman (2008) extended the concept of MSD using three different soil constitutive models: elastic perfectly-plastic, hyperbolic stress-strain curve and truncated power law in a process of energy minimisation. This allowed the displacement field to change its pattern throughout the loading sequence, and hence the early stages of loading used the 'elastic' solution and the later stages used the plastic Prandtl solution developed by Osman \& Bolton (2005).

Based on the analysis of triaxial stress-strain data available in the literature, Vardanega and Bolton (2011a) developed a power-law model to describe the non-linear stress-strain relationship of clay. This two-parameter model can be incorporated into analysis in order to get a more accurate picture of the evolution of settlement with increasing load.

\section{ANALYSIS PROCEDURE}

McMahon et al. (2013a) presents an energy method for a linear-elastic von Mises' material with associated flow. McMahon et al. (2013b) showed that this analysis can be used to investigate the optimal deformation mechanisms beneath shallow foundations both prior to and after failure. Both these papers assumed linear-elastic, perfectly plastic soil behaviour, whereas soil is well-known to behave non-linearly, even at small strains. The derivation of an appropriate $G / c_{u}$ value for use in that analysis is therefore problematic.

Vardanega and Bolton (2011a) accumulated a database of 115 triaxial and direct simple shear

Page 6 of 26 
tests on 19 natural clays, and showed that the non-linear relationship between shear stress $\tau_{m o b}$, and shear strain $\gamma=\left|\varepsilon_{1}-\varepsilon_{3}\right|$, could be approximated as:

$$
\tau_{m o b} / c_{u}=0.5\left(\gamma / \gamma_{M=2}\right)^{b} \quad \text { in the range } \quad 0.2<\tau_{m o b} / c_{u}<0.8
$$

where $\gamma_{M=2}$ is the shear strain at half the peak strength and $b$ was found to lie in the range 0.3 to 1.2 , with an average value of 0.6 and a standard deviation of 0.15 for that particular group of materials. A suite of tests on kaolin clay reported by Vardanega et al., (2012) indicated that $\gamma_{M=2}$ increased strongly with overconsolidation ratio (OCR), while $b$ increased much more gently, from about 0.4 for normally consolidated kaolin to about 0.6 at $\mathrm{OCR}=20$. Equation 2 was best suited to the range $0.2<\tau_{m o b} / c_{u}<0.8$, but for simplicity will also be deemed here to be suitable outside of this range, up to a cut-off of $\tau_{m o b} / c_{u}=1$. Figure 2 shows a comparison between the stress-strain curves of a soil following equation 2 and an equivalent elastic perfectly plastic soil with the same strain to failure. This demonstrates that extra strain energy will be absorbed by the non-linear soil, provided that $b$ is less than one. This will result in lower settlements being sustained by foundations on non-linear soil than by those on an elastic soil with the same strain to failure.

In this paper, the procedure developed by McMahon et al. (2013a) will be modified to consider the true non-linear behaviour of soil using the power-law stress-strain relationship defined by equation 2 .

\section{Non-Linear Plastic Work}

In order to carry out an upper-bound energy analysis in the style of McMahon et al. (2013a), the energy associated with deformation of a soil element must be calculated. In this paper the Page 7 of 26 
soil will be assumed to be non-linear plastic, with yield being determined by an expanding von Mises' yield surface. The von Mises' yield criterion was chosen as its circular nature in the $\pi$-plane (as opposed to the angular Tresca yield surface) makes calculation of energy dissipation simpler. The Tresca yield surface only satisfies the condition that plastic strains are parallel to total strains at the corners and the centres of the edges of the yield surface. Utilising the Tresca yield surface, while the total strain would remain in the same direction throughout the analysis, the plastic strain increment would change direction, complicating the calculation of work done.

The isotropic von Mises' yield criterion in terms of the mobilised strength in triaxial compression, $q_{m o b}$, can be expressed as:

$$
\left(\sigma_{1}-\sigma_{3}\right)^{2}+\left(\sigma_{2}-\sigma_{3}\right)^{2}+\left(\sigma_{1}-\sigma_{2}\right)^{2}=2 q_{m o b}^{2}
$$

The mobilised shear stress, $\tau_{\text {mob}}$, within the yield boundary can be determined by rearranging equation 2 , and in order to facilitate comparison with results from previously published finite element analyses, which utilised the Tresca yield criterion, it is assumed that:

$$
q_{m o b}=2 \tau_{m o b}
$$

Equations 2 and 4 can be substituted into equation 3 to determine that the von Mises' yield criterion for a soil with non-linear behaviour described by this power-law is:

$$
\left(\sigma_{1}-\sigma_{3}\right)^{2}+\left(\sigma_{2}-\sigma_{3}\right)^{2}+\left(\sigma_{1}-\sigma_{2}\right)^{2}=2 c_{u}{ }^{2}\left(\frac{\gamma}{\gamma_{M=2}}\right)^{2 b}
$$

For a von Mises' material, the total strain vector is parallel to the deviatoric stress, $\hat{\sigma}=\sigma-p$, where $p$ is the mean principal stress. As the von Mises' yield criterion is circular in the $\pi$-plane, for a material exhibiting associated flow, the direction of the incremental plastic strain vector, $\dot{\varepsilon}_{p}$, is also parallel to the deviatoric stress. If a mechanism is assumed Page 8 of 26 
that remains geometrically similar for all footing displacements, then at any given point in the mechanism the direction of the total strain vector will remain constant throughout the deformation process.

As the total strain rate in the plastic zone is equal to the plastic strain rate, it follows that:

$$
\frac{\hat{\sigma}_{2}}{\hat{\sigma}_{1}}=\frac{\dot{\varepsilon}_{2}}{\dot{\varepsilon}_{1}} \quad \text { and } \quad \frac{\hat{\sigma}_{3}}{\hat{\sigma}_{1}}=\frac{\dot{\varepsilon}_{3}}{\dot{\varepsilon}_{1}}
$$

This can be substituted, along with the condition of zero volumetric strain for undrained loading, into the yield criterion of equation 5. This produces an expression for the major principal deviatoric stress:

$$
\hat{\sigma}_{1}=\frac{c_{u} \dot{\varepsilon}_{1}}{\sqrt{3} \dot{\dot{\varepsilon}}}\left(\frac{\gamma}{\gamma_{M=2}}\right)^{b}
$$

where the strain invariant is given by:

$$
\hat{\dot{\varepsilon}}^{2}=\left[\dot{\varepsilon}_{1}^{2}+\dot{\varepsilon}_{2}^{2}+\dot{\varepsilon}_{1} \dot{\varepsilon}_{2}\right]
$$

The rate of non-linear plastic work, $\partial W_{n-l} / \partial \delta_{f}$, due to the deviatoric stress per unit volume is given by:

$$
\partial W_{n-l} / \partial \delta_{f}=\hat{\sigma}_{1} \dot{\varepsilon}_{1}+\hat{\sigma}_{2} \dot{\varepsilon}_{2}+\hat{\sigma}_{3} \dot{\varepsilon}_{3}
$$

Substitution of equations 6 and 7 into equation 9 shows the non-linear work rate per unit volume to be:

$$
\frac{\partial W_{n-l}}{\partial \delta_{f}}=\frac{2 c_{u} \hat{\dot{\varepsilon}}}{\sqrt{3}}\left(\frac{\gamma}{\gamma_{M=2}}\right)^{b}
$$

\section{Perfectly Plastic Work}

Once the mobilised shear stress given by equation 2 exceeds the undrained shear strength $c_{u}$, the soil behaves in a perfectly plastic manner. The strain at which this occurs is found from Page 9 of 26 
equation 2 to be $\gamma_{f}=\sqrt[b]{2} \gamma_{M=2}$, which is also indicated on Figure 2. For strains greater than this the soil is perfectly plastic, and the energy dissipated is thus identical to that described by McMahon et al. (2013a). The incremental work per unit volume is given by:

$$
\frac{\partial W_{p}}{\partial \delta_{f}}=\frac{4 c_{u} \hat{\dot{\varepsilon}}}{\sqrt{3}}
$$

As discussed in Klar \& Osman (2008) and McMahon et al. (2013a), the assumption of a deformation mechanism and the balancing of work and energy must, in principle, lead to an upper-bound estimate of collapse loads for perfectly rigid-plastic materials. The non-linear plastic work expressions which have been developed will now be used with a number of deformation fields to investigate the load-settlement behaviour of circular shallow foundations.

\section{DEFORMATION MECHANISMS}

\section{CAVITY EXPANSION MECHANISM}

McMahon et al. (2013a) introduced an ellipsoidal cavity expansion mechanism to describe the soil movement beneath a rough circular shallow foundation. Figure 3 illustrates notation and the global mechanism. In the near-field, ellipsoids smoothly transition from a flat punch at the ground surface to a hemisphere of radius $r_{h}$. Beyond this radius, conventional spherical cavity expansion occurs. The plastic radius, $r_{p}$, divides the fully plastic and non-linear zones of the soil and can lie in either the ellipsoidal or the spherical zone. In order to make the calculation domain finite, a bounding radius, $r_{b}$, in the spherical zone is chosen with the work done outside this radius being calculated on the basis of non-linear plastic spherical cavity expansion. This approach is valid provided that $r_{p}<r_{b}$ for the foundation settlement Page 10 of 26 
considered.

The soil movement within the hemisphere of radius $r_{h}$ is described in terms of a series of ellipsoids with a resultant soil displacement normal to the ellipsoid at each point. The first ellipsoid occurs at the footing base and hence is the special case of a circle (the footing) with normal displacement $\delta_{f}$. This corresponds to a perfectly rough footing base. Ellipsoids then grow in size and gradually transition towards being a hemisphere at $r_{h}$. Beyond the designated hemispherical boundary the soil displacements are normal to hemispheres. Hemispheres are a particular case of an ellipsoid, where $a=b=r$, and thus a similar approach can be adopted.

The deformation mechanism produced using this model is shown in Figure 4. The mechanism suffers continuity issues at the footing edge due to a gross change in geometry. Below the foundation soil displacements are purely vertical, whereas at the soil surface they are purely horizontal. An investigation demonstrated that changing the geometry at the footing edge had a negligible effect on the value of footing contact pressure for a given settlement.

\section{Non-linear plastic work beyond the bounding radius}

The principal strains for a spherical cavity expansion, $\varepsilon_{r}=2 \delta / r$ and $\varepsilon_{\theta}=-\delta / r$, can be used to find the shear strain $\gamma$.

$$
\gamma=\left|\varepsilon_{1}-\varepsilon_{3}\right|=\frac{3 \delta_{b}}{r_{b}}
$$

Compatibility, maintained by $\delta_{b}=r_{f}{ }^{2} \delta_{f} / 2 r_{b}{ }^{2}$, is combined with equation 12 and then substituted into equation 2 to determine the mobilised shear stress $\tau_{m o b}$ as:

Page 11 of 26 


$$
\tau_{m o b}=\frac{c_{u}}{2}\left(\frac{3}{2} \frac{r_{f}^{2}}{r_{b}{ }^{3}} \frac{\delta_{f}}{\gamma_{M=2}}\right)^{b}
$$

Substitution of equation 13 into the equilibrium equation and integrating between an infinite radius, $r=\infty$, where $\sigma=p_{0}$ and the bounding radius $r=r_{b}$ where $\sigma=\sigma_{b}$ produces:

$$
\int_{p_{0}}^{\sigma_{b}} d \sigma_{r}=-2 c_{u}\left(\frac{3}{2} \frac{r_{f}{ }^{2} \delta_{f}}{\gamma_{M=2}}\right)^{b} \int_{\infty}^{r_{b}} \frac{d r}{r^{3 b+1}}
$$

The in-situ stress is ignored on the grounds that gravitational effects will cancel in a work equation for the isochoric indentation of a horizontal free surface; we can therefore set $p_{0}=0$. Equation 14 can then be solved to find the stress at the bounding radius:

$$
\sigma_{b}=\frac{2 c_{u}}{3 b}\left(\frac{3}{2} \frac{r_{f}^{2}}{r_{b}{ }^{3}} \frac{\delta_{f}}{\gamma_{M=2}}\right)^{b}
$$

The work done is calculated by multiplying the stress at the bounding radius, the incremental displacement at the bounding radius and the surface area of the bounding hemisphere. This demonstrates the work beyond the bounding radius for non-linear plastic soils, $\partial W_{b, n-l} / \partial \delta_{f}$, to be:

$$
\frac{\partial W_{b, n-l}}{\partial \delta_{f}}=\frac{2 \pi}{3} \frac{c_{u} r_{f}{ }^{2}}{b}\left(\frac{3}{2} \frac{r_{f}{ }^{2}}{r_{b}{ }^{3}} \frac{\delta_{f}}{\gamma_{M=2}}\right)^{b}
$$

\section{$\underline{\text { Load-Settlement Behaviour for Cavity Expansion Mechanism }}$}

The non-linear plastic and perfectly plastic work can then be integrated over the appropriate regions within the failure mechanism, added to the work outside the bounding radius and then equated to the footing work. The relationship between footing stress and displacement for non-linear soil is hence:

$$
\sigma_{f}=\frac{1}{\pi r_{f}^{2}}\left(\frac{\partial W_{b, n-l}}{\partial \delta}+\int_{n o n-l i n e a r} \frac{\partial W_{n-l}}{\partial \delta} d V+\int_{\text {plastic }} \frac{\partial W_{p}}{\partial \delta} d V\right)
$$

Similar to the linear-elastic approach, the method of solving Equation 17 was computationally Page 12 of 26 
inexpensive because the mechanism was assumed not to change with settlement. The regions of non-linear plastic and perfectly plastic behaviour were not explicitly calculated, but the value of shear strain was simply compared to the computed value at full plasticity $\left(\gamma_{f}=\sqrt[b]{2} \gamma_{M=2}\right)$ to determine the state of a given soil region.

\section{PRANDTL MECHANISM}

Prandtl (1921) developed a mechanism for bearing failure of a strip load on the surface of a perfectly plastic material which consisted of active and passive wedge zones and a shear fan. Based on upper and lower bound plasticity analyses using this mechanism, he demonstrated that the bearing capacity factor for a strip foundation on a rigid perfectly plastic soil was $N_{c}=2+\pi=5.14$. Osman and Bolton (2005) developed a displacement field for a smooth circular footing within the boundaries of this mechanism as part of the MSD process. Figure 5 shows this displacement field.

\section{$\underline{\text { HILL MECHANISM }}$}

Hill (1949) extended the analysis of Prandtl and in doing so developed a different mechanism for a flat punch. Levin (1955) developed a displacement field within the Hill mechanism in an upper-bound investigation of the ultimate indentation pressure of smooth circular punches. This deformation field is also shown in Figure 5.

It is not possible to develop a mechanism for a rough footing within the Hill mechanism, but the smooth mechanism of Levin (1955) is used here for the purposes of comparison. 


\section{$\underline{\text { ELASTIC MECHANISM }}$}

Klar and Osman (2008) provide expressions for displacements in the lateral direction, $u$, and vertical direction, $v$, within an elastic mechanism utilising the integration of point loading solutions:

$$
\begin{aligned}
& u=\int_{0}^{2 \pi} \int_{0}^{D / 2} \frac{1}{\pi^{2}} \frac{2 z(r-\xi \cos \theta)}{\left(r^{2}+z^{2}+\xi^{2}-2 r \xi \cos \theta\right)^{3 / 2} \sqrt{D^{2}-4 \xi^{2}}} \xi \mathrm{d} \xi \mathrm{d} \theta \\
& v=\int_{0}^{2 \pi} \int_{0}^{D / 2} \frac{1}{\pi^{2}} \frac{2\left(r^{2}+2 z^{2}+\xi^{2}-2 r \xi \cos \theta\right)}{\left(r^{2}+z^{2}+\xi^{2}-2 r \xi \cos \theta\right)^{3 / 2} \sqrt{D^{2}-4 \xi^{2}}} \xi \mathrm{d} \xi \mathrm{d} \theta
\end{aligned}
$$

As the elastic displacement field extends to infinity, however, the use of an infinite series for

a displacement field was computationally expensive. An approximation to the elastic deformation field was thus generated by running a linear-elastic finite element analysis of a vertically loaded rough rigid punch in ABAQUS, with rigid boundaries at 10 foundation radii in the lateral and vertical directions. Upper-bound work calculations could hence be calculated using this displacement field within a finite domain. Bell (1991) demonstrated that finite element analyses of shallow foundations on elastic soil showed around a $10 \%$ greater stiffness with boundaries at 10 radii compared to those with boundaries at 100 radii. The calculations described here may thus underestimate the foundation displacements for any given load by a similar amount.

\section{$\underline{\text { Load-Settlement Calculations }}$}

The non-linear plastic and perfectly plastic work were integrated over the appropriate regions for Prandtl, Hill and elastic mechanisms and equated to the work done by the moving footing. The relationship between footing stress and displacement for non-linear soil is hence:

$$
\sigma_{f}=\frac{1}{\pi r_{f}^{2}}\left(\int_{\text {non-linear }} \frac{\partial W_{n-l}}{\partial \delta} d V+\int_{\text {plastic }} \frac{\partial W_{p}}{\partial \delta} d V\right)
$$

Page 14 of 26 
Again, the regions of non-linear plastic and perfectly plastic behaviour were not explicitly calculated, but the value of shear strain was simply compared to the computed value at full plasticity to determine the state of a given soil region.

\section{RESULTS}

A mesh size of $0.2 \% \mathrm{D}$ was adopted for the analysis of each mechanism. As discussed in McMahon et al. (2013a), the use of a finer mesh produced negligible changes and, therefore, did not justify the significant increase in computation time. A hemisphere of radius 2D was used for the cavity-expansion mechanism.

A parametric analysis was carried out using the cavity expansion mechanism in order to investigate the effect of the soil parameters $b$ and $\gamma_{M=2}$ on the predicted load-settlement response of the shallow foundation.

\section{Effect of $b$}

Vardanega and Bolton (2011a) suggest that $b$ has an average value of 0.6 and a standard deviation of 0.15 . For $b$ values of $0.45,0.6$ and 0.75 , the load-settlement curve for $\gamma_{M=2}=0.5 \%$ is given in Figure $6(\mathrm{a})$ and that for $\gamma_{M=2}=1 \%$ in Figure $6(\mathrm{~b})$. The effect of a change in $b$ value from 0.45 to 0.6 can be seen to be greater than that for a change from 0.6 to 0.75. Vardanega and Bolton (2011a) found from their database that the extreme values of $b$ were 0.3 to 1.2 . It is argued that $b$ should not be greater than 1 as this corresponds to a stress-strain curve with an increasing rate of strain-hardening up to its ultimate plastic 
strength. Only two tests appear to have $b$ values greater than 1 which, from examination of the source data, could well have been affected by errors in digitisation of the original data. By definition, $68 \%$ of data lies within one standard deviation of the mean, and it is shown in Figure 6 that if the average value is adopted then no significant error should occur for the majority of soils. Vardanega and Bolton (2011a) investigated the dependence of $b$ on other parameters and found it to be most likely a function of the soil structure, fabric, presence of fissures and the general sample condition. Of course, with any real soil a laboratory test will provide a value of $b$ and this is the value that should be used in any analysis. For demonstrative purposes the average value of $b=0.6$ is adopted where necessary.

\section{Effect of $\gamma_{M=2}$}

Using a value of $b=0.6$ allows load-settlement curves for soils with different values of $\gamma_{M=2}$ to be determined. The result is shown for 4 different soils in Figure 7. The particular values of $\gamma_{M=2}$ were chosen based on results published for kaolin clay in Vardanega et al. (2012). With specific soil data an empirical expression for the reference strain $\gamma_{M=2}$ was found as a function of the overconsolidation ratio (OCR) of kaolin. This was determined to be:

$$
\gamma_{M=2}=0.0040(O C R)^{0.680}
$$

The values in Figure 7 correspond to a normally consolidated soil and soils with OCRs of 5, 10 and 20. It can be seen that the use of a fixed factor of safety for all soils implies very different tolerable settlements for foundations on soils with different OCR. For example, the use of a factor of safety of 3 implies tolerable settlements of $0.1 \%$ of a diameter on normally consolidated soil, but $0.7 \%$ of a diameter on soil with an OCR of 20 . While the use of a factor of safety does limit settlements and maintain serviceability, the use of an arbitrary value for Page 16 of 26 
all soils may be overly conservative for low OCR soils.

\section{MECHANISM COMPARISON}

A parametric analysis was performed for a number of values of $\gamma_{M=2}$ for $b$ values of $0.45,0.6$ and 0.75 . It was found that for each value of $b$ a single design line could be recommended when the normalised footing pressure $\sigma_{f} / c_{u}$ was plotted against $\delta /\left(D \gamma_{M=2}\right)$ on log-log axes. Figures 8(a), (b) and (c) show the strain-hardening stress-displacement results for all four displacement mechanisms - cavity expansion, Prandtl, Hill and linear elasticity - for $b$ values of $0.45,0.6$ and 0.75 respectively, together with the classical plastic solution of Eason and Shield (1960). The changes in $b$ value are not very significant for the cavity expansion, Prandtl and Hill mechanisms. The elastic mechanism, however, shows a significant change across the three values of $b$, with a factor of 2 difference in the settlement.

\section{Implications for settlement prediction}

McMahon et al. (2013b) performed a similar comparison for a linear-elastic perfectly plastic material and demonstrated that the elastic displacement mechanism was optimal in the small strain range, followed by the cavity expansion mechanism up to approximately the classical value of bearing capacity, beyond which the Prandtl mechanisms was optimal. Taking the particular value of $b=0.6$, shown in Figure 8(b), the elastic mechanism and cavity expansion mechanisms are very similar in the small settlement region up to $\delta /\left(D \gamma_{M=2}\right) \approx 0.4$; from this value up to $\delta /\left(D \gamma_{M=2}\right) \approx 5$, the cavity expansion mechanism provides the lowest upper-bound solution, beyond which the classical bearing capacity value $\sigma_{f} / c_{u} \approx 6$ is then most appropriate. The Prandtl mechanism is shown to reach an ultimate value of $N_{c}=6.11$. The 
Hill mechanism is slightly higher, obtaining a value of $N_{c}=6.16$. Although these mechanisms correspond to smooth footings, the results are similar to the value found by Eason and Shield (1960) for a rough circular foundation.

Each of the mechanisms provided a plot of normalised bearing pressure versus normalised settlement that began as straight lines on a log-log plot, echoing the power law which was used as the soil constitutive model. For the values of $b$ that were investigated, the optimal (lowest) of these mechanistic upper-bound calculations was provided either by the cavity expansion mechanism or the elastic mechanism, except that the cavity expansion mechanism always gave the most reasonable asymptotic approach to failure.

For decision-making purposes, the correct approach is simply to select the interior envelope created by the various mechanistic trials. Figure 9 displays these envelopes for the three selected values of power exponent $b$. Two aspects of these envelopes are significant. First, they are effectively straight for small displacements, and their slopes are identical to $b$, which should have been considered mathematically inevitable. Secondly, the three linear approximations, also shown in Figure 9, effectively converge on the point $\delta /\left(D \gamma_{M=2}\right)=2$, $\sigma_{f} / c_{u}=6$. For small to moderate strength mobilisations, $\sigma_{f} / c_{u} \leq 4$, the deviation in calculated settlement between the linearized and calculated envelopes amounts to less than $20 \%$. Few shallow foundations will be designed with safety factor $F \approx 6 c_{u} / \sigma_{f}<1.5$, so settlements in service can now be estimated from the linear approximations as follows.

$$
\log 6-\log \frac{\sigma_{f}}{c_{u}}=b\left[\log 2-\log \frac{\delta}{D \gamma_{M=2}}\right]
$$




$$
\begin{aligned}
& \frac{\delta}{2 D \gamma_{M=2}} \approx\left(\frac{\sigma_{f}}{6 c_{u}}\right)^{1 / b} \\
& \frac{\delta}{D} \approx \frac{2 \gamma_{M=2}}{F^{1 / b}} \quad \text { for } 1.5<F<5
\end{aligned}
$$

recalling that Vardanega \& Bolton (2011a) placed limits $1.25<F<5$ on the reliable use of the power law model itself.

Equation 21 can be shown to emulate the form of the original MSD solution of Osman \& Bolton (2005) if the power law is used for the soil stress-strain relation. They postulated that the average soil strength mobilised immediately beneath the foundation could be taken as $\sigma_{f} / N_{c} \approx \sigma_{f} / 6$, and they estimated the average shear strain $\gamma$ within the same local region, lying within the boundaries of a plane strain Prandtl bearing capacity solution, as $1.35 \delta / D$. They proposed to link these estimates through the stress-strain relation of the soil. So using equation 2, we find:

$$
\begin{aligned}
& \frac{\sigma_{f}}{c_{u}}=0.5\left[\frac{1.35 \delta}{D \gamma_{M=2}}\right]^{b}=\frac{1}{F} \\
& \frac{\delta}{D} \approx \frac{2^{1 / b} \gamma_{M=2}}{1.35 F^{1 / b}}
\end{aligned}
$$

Osman \& Bolton (2005) showed, for a soil profile modelling London clay, that equation 22 could accurately fit a non-linear finite element simulation of the loading of a circular foundation based on the soil properties at a relative depth of $0.3 D$. A stress-strain function was fitted to the data of high-quality samples of London clay. A later interpretation of a larger database showed that such data fitted a power law with $\mathrm{b}=0.58$ : Vardanega \& Bolton (2011b). Substituting into equation 22 gives: 


$$
\frac{\delta}{D} \approx \frac{2.45 \gamma_{M=2}}{F^{1 / b}}
$$

Although this is $22 \%$ greater than the estimate following the current work, in equation 21 , it may be noted that an exact match between equation 21 and the FE analysis of Osman \& Bolton (2005) could still be obtained if the representative depth of a soil sample were simply shifted slightly to $0.35 D$.

It must not be forgotten, of course, that the calculation of settlement following equation 21 utilises the value of safety factor $F$, and therefore depends on the selection of a representative value of strength $q_{u}$ in the von Mises' equation 3, from which the shear strength $c_{u}$ is derived here according to equation 4. An initial estimate might be made, following the discussion of anisotropy in Osman \& Bolton (2005), by using the average of the triaxial strengths in compression and extension on samples taken from the representative depth. However, a more rigorous analysis would clearly have to involve FE analysis with an anisotropic failure criterion, and an initial earth pressure coefficient $K_{0}$ based on site measurements.

\section{IMPLICATIONS FOR MSD METHODOLOGY}

The semi-intuitive approach of Osman \& Bolton (2005) used a kinematically acceptable displacement field based on the Prandtl mechanism to provide reasonable solutions for the settlement of a shallow circular foundation on clay by linking mobilised stresses and strains immediately beneath the foundation. However, the current work confirms the finding of Klar \& Osman (2008) that a more rigorous application of energy principles shows that a shallow, local mechanism, either following Hill or Prandtl, does not offer the optimum (lowest) upper bound load-displacement relation. Instead, either the new cavity expansion mechanism Page 20 of 26 
proposed by McMahon et al. (2013a), or a displacement field based on linear elastic solutions, provides a better (greater) estimate of non-linear displacements when solved using conservation of energy. When a more exhaustive envelope to the normalised load-displacement curve was obtained, it was demonstrated in equation 21 that a functionally similar result to Osman \& Bolton (2005) was obtained for moderate strength mobilisations, even though the latter had invoked a less critical mechanism in their derivation. Equation 21 offers designers a very simple way to estimate settlements on undrained clay with typical non-linear behaviour, based on fitting a power curve to triaxial test data from a representative sample in order to estimate parameters $b$ and $\gamma_{M=2}$.

In principle, either MSD or FE analysis could be used to extend equation 21 by introducing different $\mathrm{K}_{0}$ values and various rates of strength increasing with depth, for example. It should be expected, following the foregoing analysis, that any corrections would be in the form of alterations to the factor 2 derived in equation 21, so long as the stress-strain relation of the clay continues to be well-modelled by a power curve.

\section{CONCLUSIONS}

The energy method for a linear-elastic perfectly-plastic soil employing the von Mises' yield criterion with associated flow, as developed by McMahon et al. (2013a), was extended to consider the behaviour of non-linear plastic soil. Expressions for the non-linear plastic work done in deformation were developed. This non-linear approach was used with the ellipsoidal cavity-expansion mechanism developed by McMahon et al. (2013a), the classical Prandtl and Hill mechanisms, and an elastic mechanism obtained through finite element analysis, to 
calculate load-settlement plots for shallow foundations for each of these deformation mechanisms.

The power curve representation of the stress-strain relationship of clays was adopted, following Vardanega \& Bolton (2011a), with exponent $b$ and mobilization strain $\gamma_{M=2}$. The influence of $b$ was investigated and it was shown that only small differences arise in the non-linear settlement solutions obtained from cavity expansion, Hill and Prandtl mechanisms when using $b$ values in the common range $0.6 \pm 0.15$. Laboratory testing of site-specific soil would, of course, provide the most appropriate value for use in analysis.

A parametric analysis provided a single design line when the normalised footing pressure $\sigma_{f} / c_{u}$ was plotted against normalized settlement $\delta /\left(D \gamma_{M=2}\right)$. For the typical value of $b=0.6$ the cavity-expansion mechanism provides a better upper-bound solution for footing settlement than the Prandtl \& Hill mechanisms up to $\sigma_{f} / c_{u} \approx 6$, close to the classical value of bearing capacity for a rough foundation. Beyond this value, the Prandtl mechanism is more appropriate. Since the power curve expression of Vardanega \& Bolton (2011a) was specifically fitted to $0.2<\tau / c_{u}<0.8$, it must follow that the non-linear settlement curves derived here should not be expected to apply accurately outside the bounds $1.2<\sigma_{f} / c_{u}<4.8$. Furthermore, a single power-law expression was derived, equation 21, by which the settlement of a shallow foundation could conveniently be estimated within $20 \%$ bounds for normalized bearing stresses in the range $1.2<\sigma_{f} / c_{u}<4$. 


\section{ACKNOWLEDGEMENTS}

The first author would like to thank the financial support he received from the Cambridge Australia Trust (Poynton Scholarship) and the Principals of UK Universities (Overseas Research Students Awards Scheme) throughout his studies in Cambridge. 


\section{REFERENCES}

Bell, R. W. (1991) The analysis of offshore foundations subjected to combined loading. MSc Thesis, Oxford University, UK

Bolton, M. D. (2013). Performance-based design in geotechnical engineering. Rankine Lecture, Géotechnique (in press)

Burland, J. B. (1989). 'Small is beautiful': the stiffness of soils at small strains. $9^{\text {th }}$ Laurits Bjerrum Memorial Lecture, Canadian Geotechnical Journal, 26, No. 4, 449-516.

Eason, G. and Shield, R. T. (1960). The plastic indentation of a semi-infinite solid by a perfectly rough circular punch. ZAMP, 11, 33-43.

Hill, R. (1949). The plastic yielding of notched bars under tension. The Quarterly Journal of Mechanics \& Applied Mathematics, 2, No.1, 40-52.

Houlsby, G. T. and Wroth, C. P. (1991). Variation of shear modulus of a clay with pressure and overconsolidation ratio. Soils and Foundations, 31, No. 3, 138-143.

Jardine, R. J., Symes, M. J. and Burland, J. B. (1984). The measurement of soil stiffness in the triaxial apparatus. Géotechnique, 34, No. 3, 323-340.

Klar, A. and Osman, A. S. (2008). Load-displacement solutions for piles and shallow foundations based on deformation fields and energy conservation. Géotechnique 58, No. $7,581-589$.

Levin, E. (1955). Indentation pressure of a smooth circular punch. Quarterly of Applied Mathematics, 13, No. 2, 133-137.

Mair, R. J. (1993). Developments in geotechnical engineering: application to tunnels and deep excavations. Proceedings of the Institution of Civil Engineers, Civil Engineering, 93, 27-41.

Page 24 of 26 
McMahon, B. T., Haigh, S. K. and Bolton, M. D. (2013a). Cavity expansion model for the bearing capacity and settlement of circular shallow foundations on clay. Géotechnique. 63, No.9 746-752

McMahon, B. T., Haigh, S. K. and Bolton, M. D. (2013b). Optimal displacement mechanisms beneath shallow foundations on linear-elastic perfectly plastic soil. Géotechnique 63, No. $16,1447-1450$

Osman, A. S. and Bolton, M. D. (2005). Simple plasticity-based prediction of the undrained settlement of shallow circular foundations on clay. Géotechnique 55, No. 6, 435-447.

Prandtl, L. (1921). Über die Eindringungsfestigkeit Plastischer Baustoffe und die Festigkeit von Schneiden. Zeitschrift für Angewandte Mathematik und Mechanik, 1, No. 1, 15-20.

Vardanega, P. J. \& Bolton, M. D. (2011a). Strength mobilization in clays and silts, Canadian Geotechnical Journal, 48, No. 10, 1485-1503.

Vardanega, P. J. \& Bolton, M. D. (2011b). Predicting shear strength mobilization of London clay, Proceedings of the 15th European Conference on Soil Mechanics and Geotechnical Engineering, Athens, 487-492.

Vardanega, P. J. \& Bolton, M. D. (2012). Corrigendum, Canadian Geotechnical Journal, 49, No. 5,631 .

Vardanega, P. J. Lau, B. H., Lam, S. Y., Haigh, S. K., Madabhushi, S. P. G. \& Bolton, M. D. (2012). Laboratory measurement of strength mobilisation in kaolin: link to stress history. Géotechnique Letters, 2, 9-15. 


\section{$\underline{\text { List of Figures }}$}

Figure 1: Stiffness versus strain and typical strain ranges for structures (redrawn from Mair, 1993)

Figure 2: Non-linear stress-strain curve

Figure 3: Notation for the ellipsoidal cavity expansion mechanism

Figure 4: Ellipsoidal cavity expansion mechanism

Figure 5: Displacement fields of Levin (1955) within the Hill mechanism and Osman and Bolton (2005) within the Prandtl mechanism

Figure 6: Effect of the parameter $b$ on the load-settlement behaviour

a) Load-settlement behaviour for $\gamma_{M=2}=\mathbf{0 . 0 0 5}$

b) Load-settlement behaviour for $\gamma_{M=2}=0.01$

Figure 7: Load-settlement behaviour of clays $(b=0.6)$ for different characteristic strain $\left(\gamma_{M=2}\right)$ values with a corresponding OCR for kaolin clays determined using the correlation of Vardanega et al. (2012)

Figure 8: Comparison of the mechanisms for the non-linear soil model with:
a) $b=0.45$
b) $b=0.6$
c) $b=0.75$

Figure 9: Best (lowest) upper-bound solutions for all $b$-values 


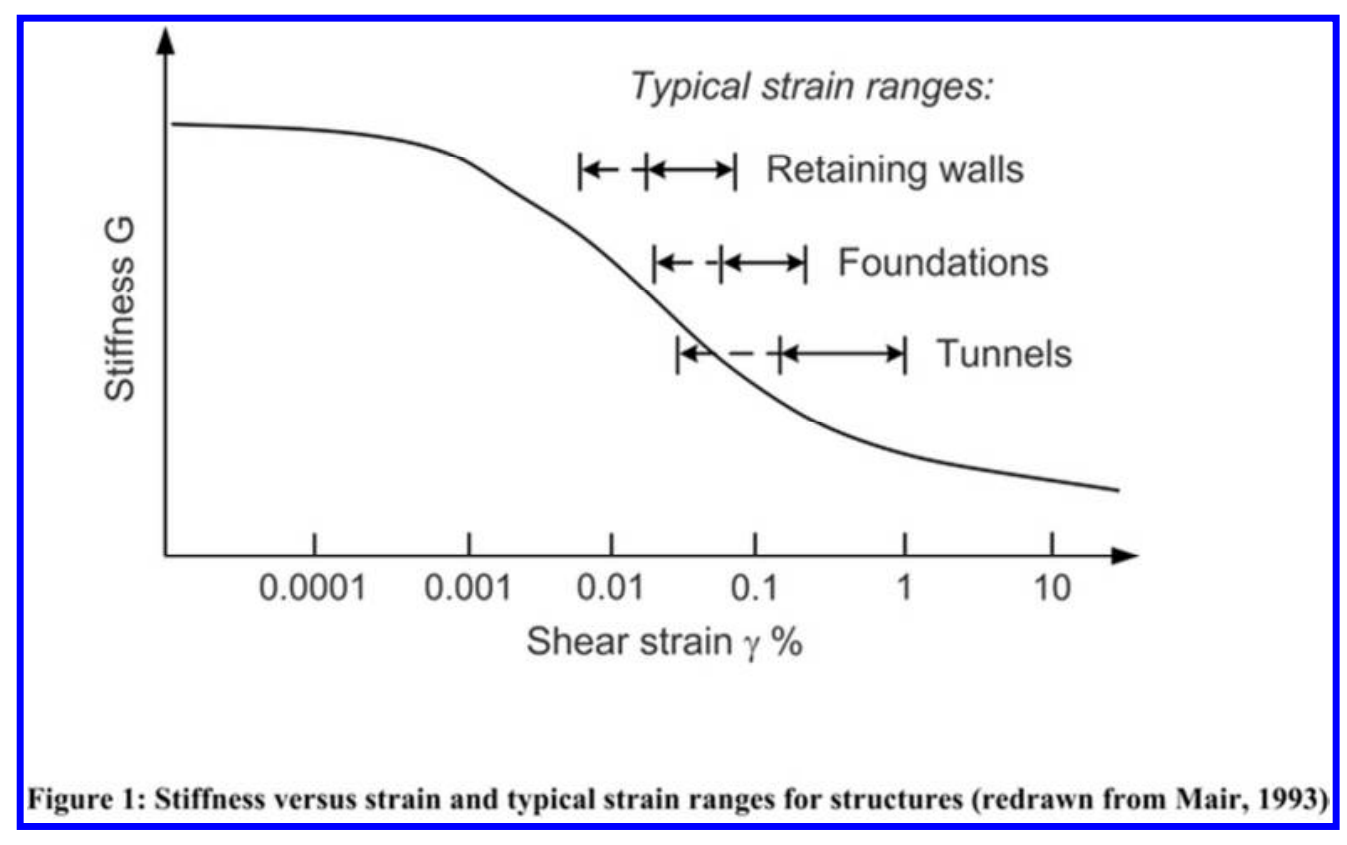

$54 \times 33 \mathrm{~mm}(300 \times 300$ DPI $)$ 


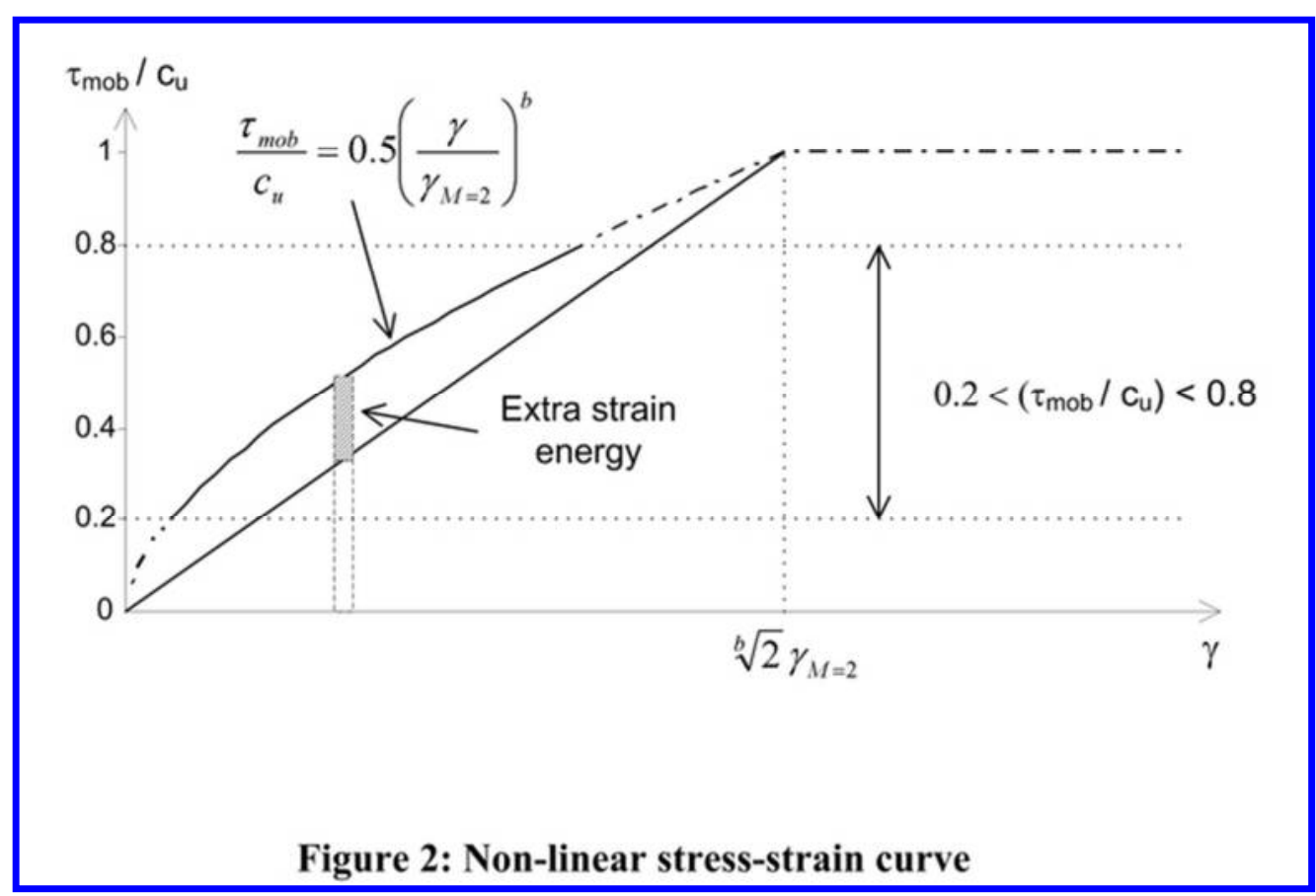

$59 \times 39 m m(300 \times 300$ DPI $)$ 


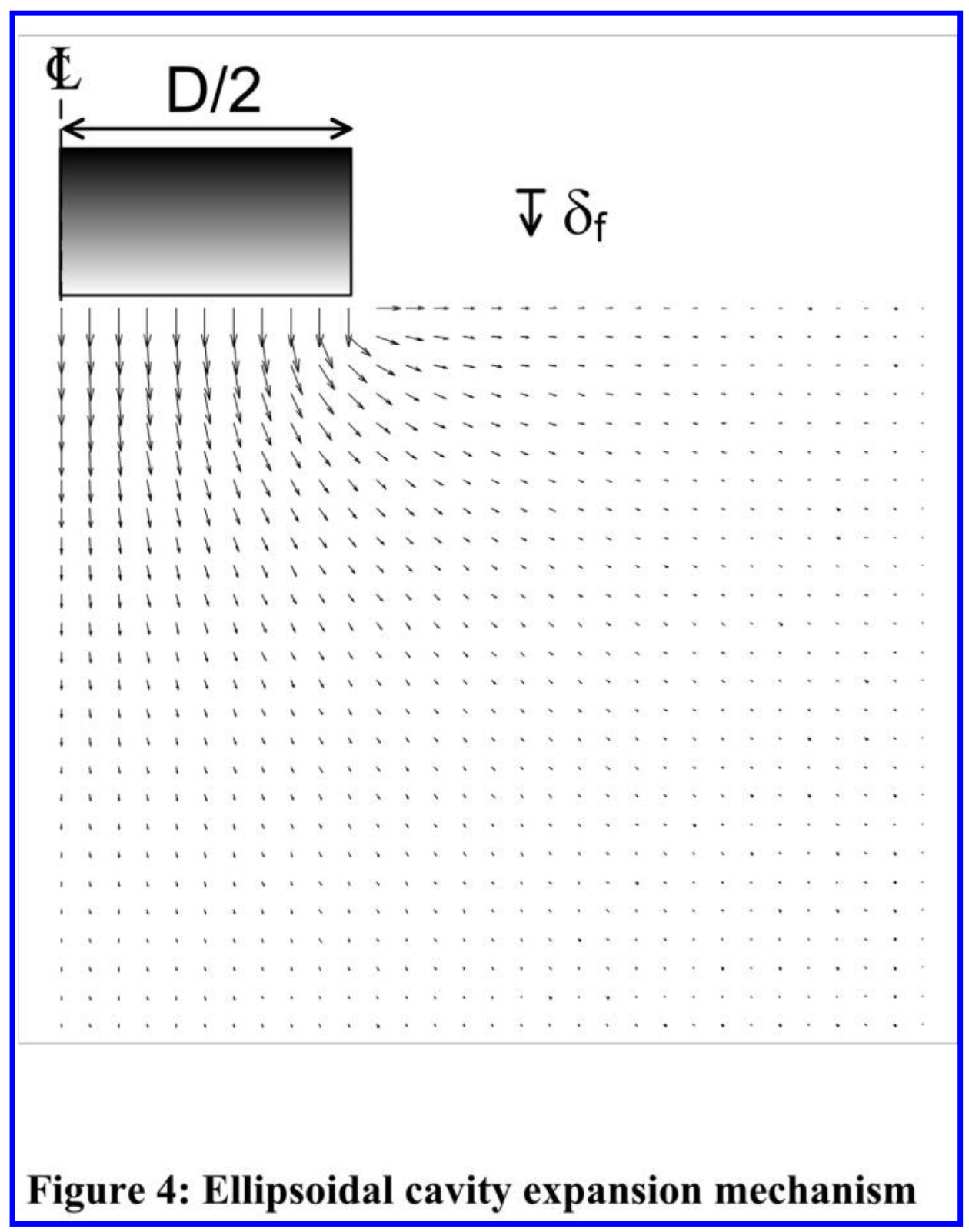

$115 \times 147 \mathrm{~mm}(300 \times 300$ DPI $)$ 


\section{Page 31 of 35}

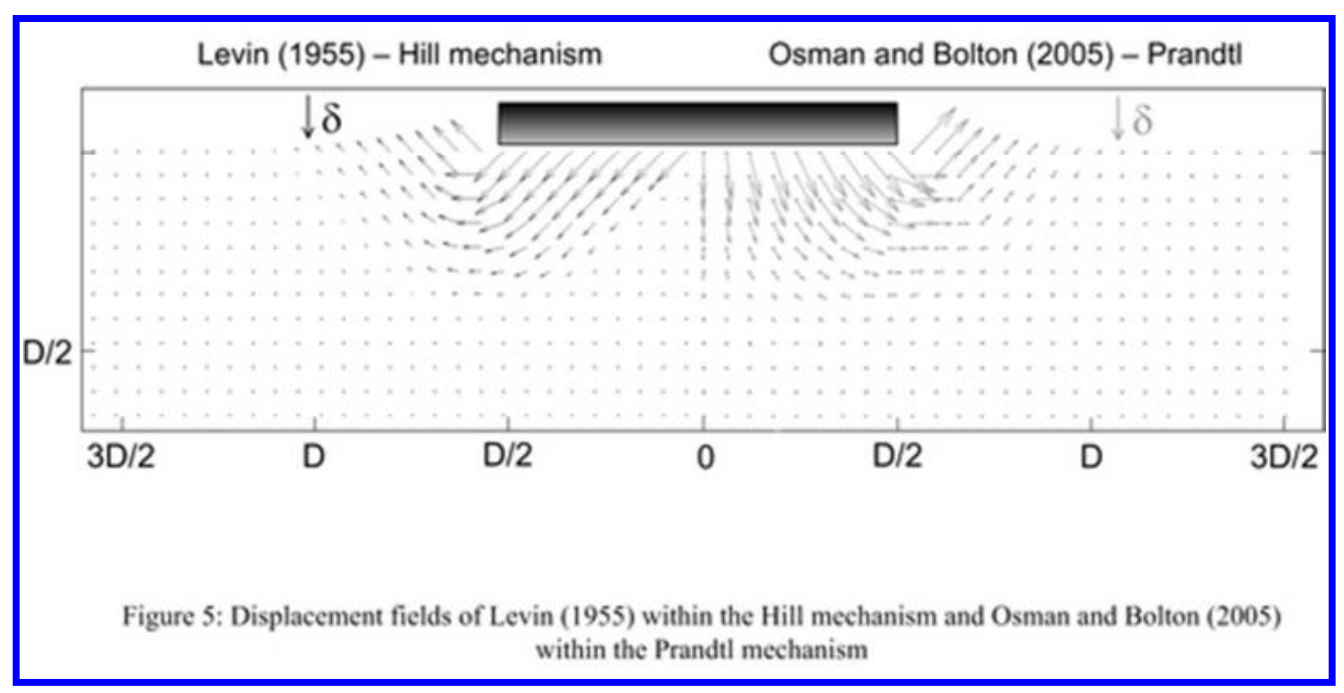

$45 \times 22 \mathrm{~mm}(300 \times 300 \mathrm{DPI})$ 


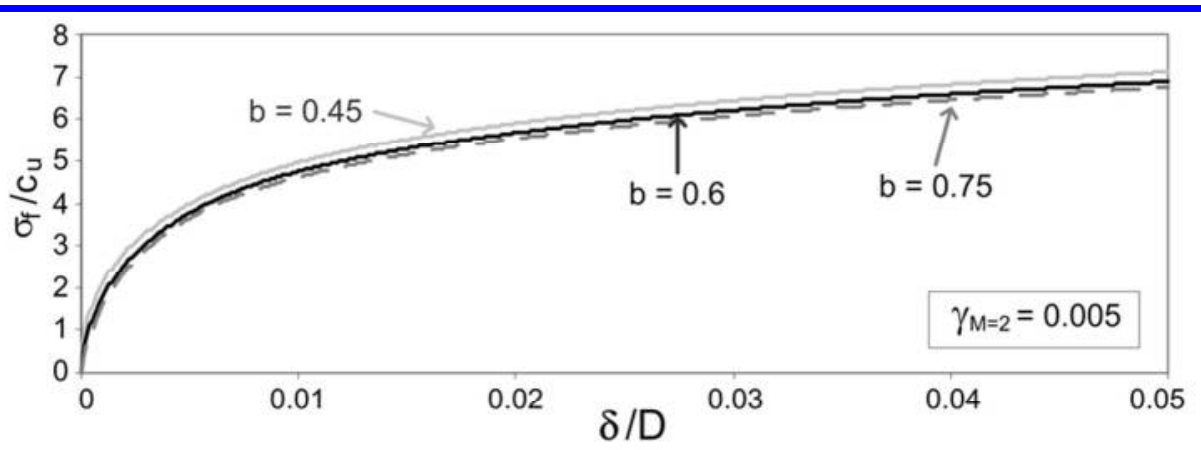

(a)

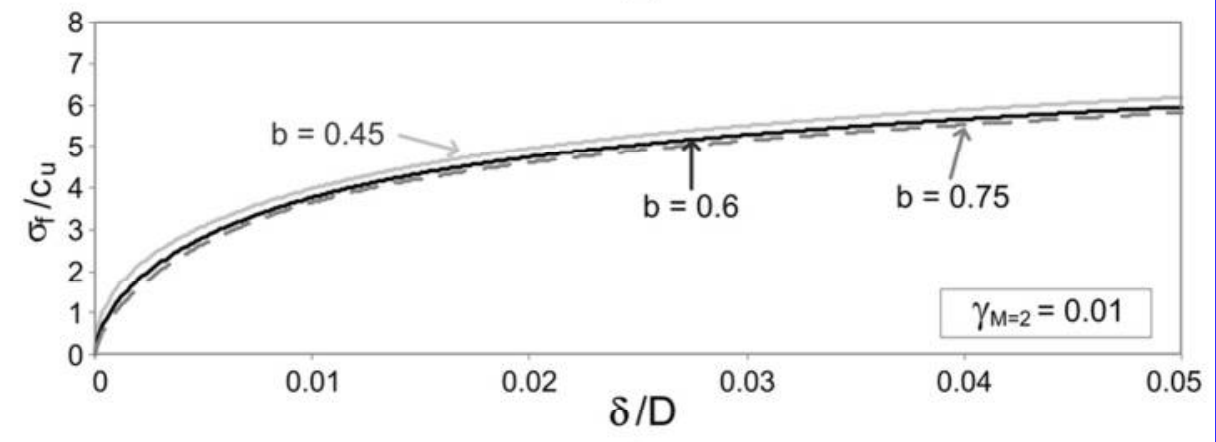

(b)

Figure 6: Effect of the parameter $b$ on the load-settlement behaviour

a) Load-settlement behaviour for $\gamma_{M=2}=\mathbf{0 . 0 0 5}$

b) Load-settlement behaviour for $\gamma_{M=2}=0.01$

$80 \times 71 \mathrm{~mm}(300 \times 300$ DPI $)$ 


\section{Page 33 of 35}

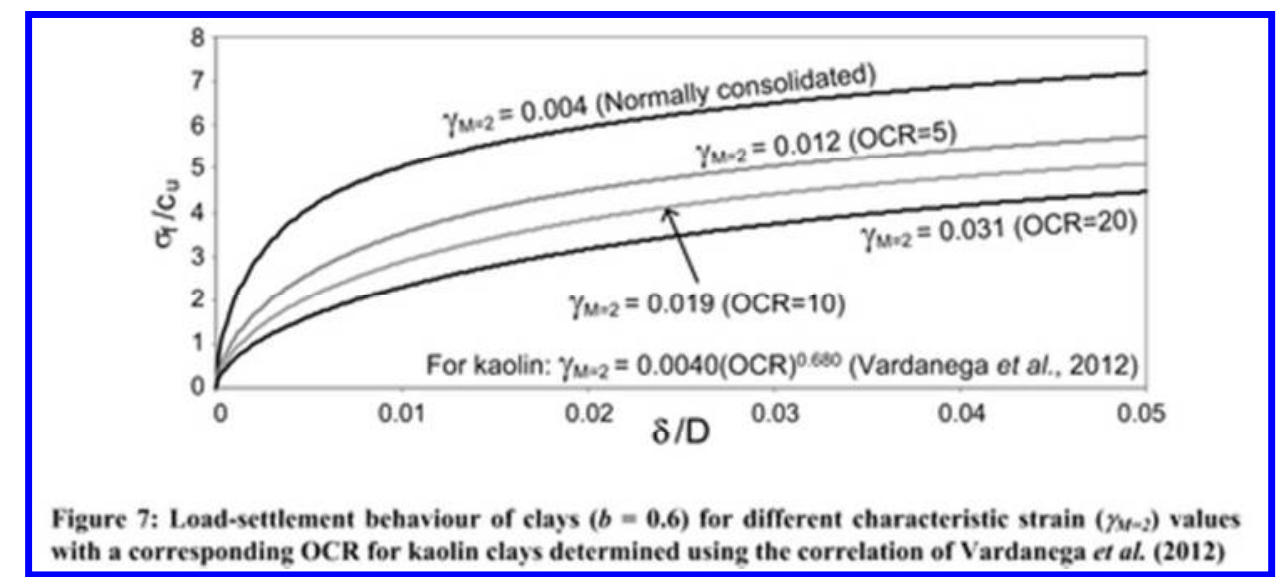

$39 \times 17 \mathrm{~mm}(300 \times 300 \mathrm{DPI})$ 
(a)

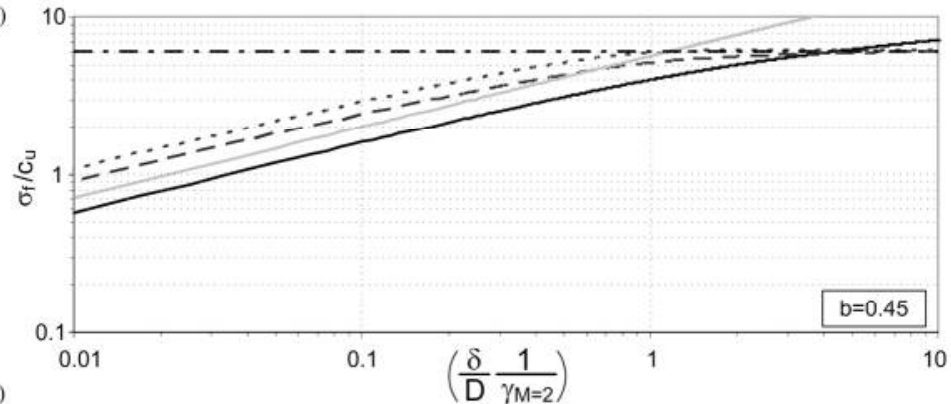

(b)
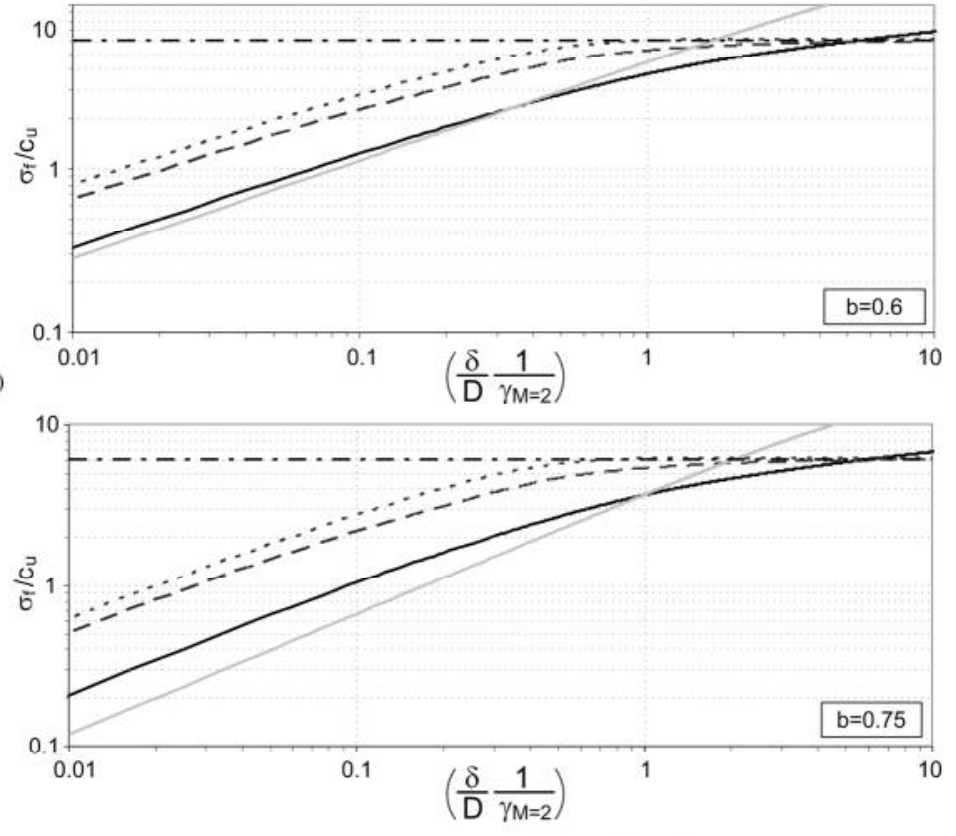

Rough Footing Mechanisms

Smooth Footing

Elastic

- McMahon et al. (2013) - Cavity expansion Mechanisms

-..-- Eason \& Shield (1960)

-. Levin (1955) - Hill

Figure 8: Comparison of the mechanisms for the non-linear soil model with: (a) $b=0.45$ (b) $b=0.6$ (c) $b=0.75$

$134 \times 202 \mathrm{~mm}(300 \times 300$ DPI $)$ 


\section{Page 35 of 35}

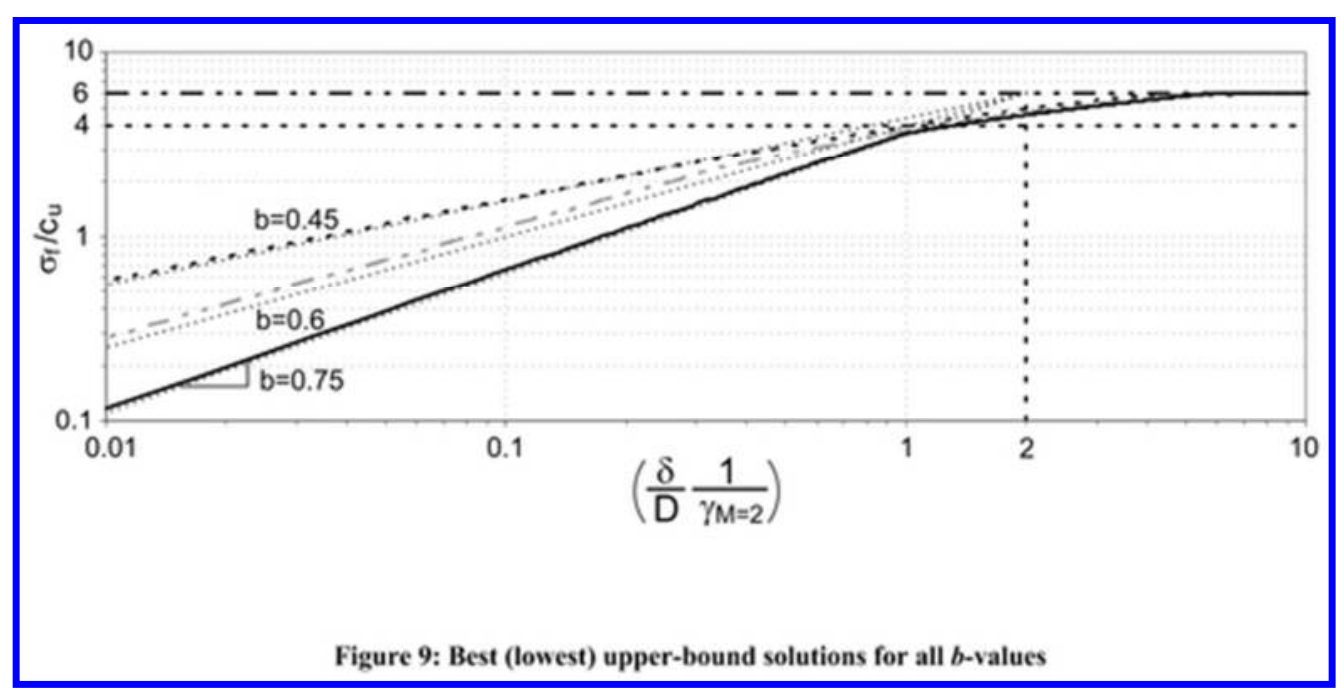

$44 \times 22 \mathrm{~mm}(300 \times 300$ DPI $)$ 